\title{
Transfer of inhibition and facilitation mediated by the original target stimulus
}

\author{
ROBERT A. RESCORLA \\ University of Pennsylvania, Philadelphia, Pennsylvania
}

\begin{abstract}
An autoshaping paradigm with pigeon subjects was used to study two types of modulation: facilitation and inhibition. In each paradigm, a diffuse stimulus signaled when a keylight would be reinforced. Transfer of that diffuse stimulus to a different keylight was assessed after the responseevoking power of the original keylight had been altered in the presence of a different modulator. That alteration of the original target produced a specific change in the transfer of modulators trained with that target. The results are interpreted as suggesting that transfer to another target stimulus is partly mediated by the association of the original target with the reinforcer. They are inconsistent with at least one version of a configural cue interpretation.
\end{abstract}

It is now clear that various Pavlovian conditioning paradigms can generate stimuli whose primary function is to modulate the associations of other stimuli with an unconditioned stimulus (US). Such modulators can serve either a facilitative function, setting the occasion on which another stimulus will be followed by the US, or an inhibitory function, signaling occasions on which another stimulus will not be followed by the US.

For instance, in a conditioned inhibition, or negative occasion setting, design, an animal is presented with two kinds of trials: $A+$ trials, on which $A$ is followed by a US, and XA- trials, on which A is accompanied by $X$ but the US is omitted. Quite a number of experiments suggest that, under some circumstances, this training results in an A-US association that is modulated by $X$. Similarly, in the converse facilitation, or positive occasion setting, design, the animal receives trials on which $A$ is presented alone without the US (A-) as well as trials on which $A$ is accompanied by $X$ and then followed by the US (XA+). In extensive series of experiments, Holland (e.g., Holland, $1983,1985,1989 a)$ and Rescorla $(1985,1986 b)$ have found that such a procedure commonly endows A with a direct excitatory association with the US while conferring on $\mathrm{X}$ the ability to modulate positively the strength of that A-US association.

One issue of theoretical interest for both positive and negative modulators has been the degree to which they transfer their power to other stimuli. That issue is significant not only for purposes of determining the range of importance of modulators but also for characterizing their mode of action. It is now clear that under a broad range of circumstances, an $\mathrm{X}$ trained either as a facilitator or as an inhibitor will modulate not only the A with

\footnotetext{
This research was supported by National Science Foundation Grants BNS 83-08176 and BNS-88-03514. Correspondence concerning this article should be addressed to Robert A. Rescorla, Department of Psychology, University of Pennsylvania, 3815 Walnut St., Philadelphia, PA 19104.
}

which it was trained but also another stimulus that is perceptually distinct but treated in the same manner as A (e.g., Holland, 1989a, 1989b, 1989c; Rescorla, 1985). That successful transfer suggests a locus of action for the modulator that is not specific to the A stimulus or the A-US association. One reasonable possibility is that the modulator acts on a representation of the US, perhaps by raising its threshold for activation (Rescorla, 1985). Nevertheless, under many conditions, transfer of X's modulation to a B stimulus is incomplete even when B has been trained with the same US under conditions that closely match the training of A (e.g., Holland, 1989a; Rescorla, 1985). That incompleteness of the transfer suggests a certain specificity of X's action either to the A or to the A-US association.

There might be many bases for this specificity. For instance, some of the learning in modulatory paradigms might involve configurational processes. The joint occurrence of $A$ and $X$ might result in an emergent stimulus not well characterized as the addition of $\mathrm{X}$ to $\mathrm{A}$. To the degree that such a configural stimulus serves as the basis of a discrimination between $\mathrm{AX}$ and $\mathrm{A}$, then the effect of $X$ might not fully transfer to $B$ because of the dissimilarity between AX and BX. Although configural cues are unlikely to serve as the sole basis of modulation in such paradigms, a partial contribution could produce specificity of action (Holland, 1985). Another possibility is that part of the manner in which $X$ acts is to modulate responding to the particular A stimulus or the A-US association with which it was trained. According to such a view, transfer might be partially based on generalization between either A and B or between the A-US and B-US associations.

The experiments reported here derived from the second of these possibilities. They investigated one implication of the view that X's effect on B is partially mediated by the A-US association with which $\mathrm{X}$ was originally trained. If that view is correct, then subsequent manipulations of the A-US association might affect the degree to which $\mathrm{X}$ transfers its modulatory effect to $\mathrm{B}$. This pos- 
sibility was investigated in two experiments, one using a conditioned inhibition paradigm and one using a conditioned facilitation paradigm. In each experiment, two modulators were established with separate targets and then tested for their transfer to another common target. However, prior to that transfer test, the status of the original target-US association was altered for one modulator, but not for the other. In each case, the question was how that alteration in the status of the original target would affect transfer of modulation.

Both experiments involved standard autoshaping procedures in pigeon subjects, using keylights as signals of a food US and diffuse auditory and visual stimuli as the modulators. In such procedures, which have been extensively used for the study of modulators, the keylights come to elicit directed pecking and the diffuse stimuli modulate that pecking.

\section{EXPERIMENT 1}

This experiment examined the impact on the transfer of a facilitator produced by manipulation of the original target of that facilitator. As illustrated in Figure 1, birds were concurrently trained in two facilitation paradigms, each having a localized keylight (blue or a pattern), which was followed by food only in the presence of a diffuse auditory facilitator (noise or tone). In addition, a diffuse light (L) served as a facilitator for each keylight. Then, an attempt was made to reduce the association of one of the keylights with food. It seemed unlikely that separate nonreinforced presentations of the keylight would accomplish this, since they were already an inherent part of the facilitation training procedure. Hence, reduction in the target-US association was accomplished by repeated nonreinforced presentation of the target during the light (cf. Rescorla, 1986a). After that treatment of one of the original target keylights, a transfer keylight (white) was prepared in the manner of previous experiments (e.g., Rescorla, 1985) by training and extinction. Finally, both diffuse auditory stimuli were tested for transfer to that target. To the degree that the auditory facilitators transfer independently of their own original targets, they should show comparable transfer. However, to the degree that transfer of facilitation is partially mediated by the origi-

\begin{tabular}{|c|c|c|c|}
\hline Training & Ext & Target & Test \\
\hline $\mathrm{NP}+, \mathrm{P}-, \mathrm{LP}+$ & LP- & \multirow{2}{*}{$w+/-$} & \multirow{2}{*}{$W-. T W-. N W-$} \\
\hline $\mathrm{TB}+, \mathrm{B}-, \mathrm{LB}+$ & LB- & & \\
\hline
\end{tabular}

Figure 1. Design of Experiment 1. (L, N, and $T$ are diffuse 15sec light, noise, and tone, respectively. P (pattern) and B (blue) are the original training keylights, whereas white $(W)$ is the target keylight for transfer. Presentation and omission of food are indicated by + and - , respectively. (For expositional purposes, only half of the counterbalancing of stimuli is displayed; $P$ and $B$ were interchanged for half of the animals.) nal target, the facilitator whose target has been treated should show reduced transfer.

\section{Method}

Subjects and Apparatus. The subjects were 16 female Carneau pigeons about 1 year old. They had participated in another autoshaping experiment that had used different stimuli and a different response key. The assignment of birds to groups in the present experiment was random with respect to their previous treatments. They were housed in pairs and maintained at $80 \%$ of their free-feeding weights.

The apparatus was made up of eight identical operant chambers, each measuring $27 \times 27 \times 35 \mathrm{~cm}$. The metal front panel of each chamber had a $5 \times 5 \mathrm{~cm}$ food magazine in its center, located $5 \mathrm{~cm}$ above the wire mesh floor. Three response keys, $2.5 \mathrm{~cm}$ in diameter, were located one directly above the hopper and one on each side of the center of the front wall, $20 \mathrm{~cm}$ above the floor. Located behind the right-hand key was an IEE inline projector that permitted the transillumination of the key with three light stimuli: blue (B), white $(W)$, and a grid pattern (P) made up of $1-\mathrm{mm}$ black lines spaced $2 \mathrm{~mm}$ apart and oriented $45^{\circ}$ from the vertical on an otherwise white background. The blue light was confined to the bottom portion of the response key; the grid pattern and white were confined to the top portion of the key. The other half of the key remained unilluminated in each case. These stimuli were generated by Ektachrome slides of drawings composed of "Color-aid" artist paper.

The other three walls and ceiling of each of the chambers were composed of clear Plexiglas. These chambers were placed in soundand light-attenuating shells, with ventilation fans providing background noise of $62 \mathrm{~dB}$ re $20 \mathrm{uN} / \mathrm{m}$. On the rear wall of those shells was mounted a $6-\mathrm{W}$ bulb that was continuously illuminated during the session, except during the operation of the food hopper, which contained Purina Pigeon Grain. That houselight could be interrupted at the rate of $2 / \mathrm{sec}$ to provide a diffuse visual signal (L). Also mounted on that wall were two speakers that allowed the presentation of a white noise $(\mathrm{N})$ and an $1800-\mathrm{Hz}$ tone $(\mathrm{T})$, each of which raised the sound level to about $80 \mathrm{~dB}$.

Experimental events were automatically controlled by relay equipment and microprocessors located in an adjoining room.

Procedure. Because of the birds' past experience in an autoshaping experiment, no magazine training was necessary. On the first 2 days of the experiment, they received a pretest of the two keylights to be used as targets in the original training. On each day, they received 18 nonreinforced 5-sec presentations each of $B$ and $P$. During this and all subsequent phases of the experiment, the intertrial interval (ITI) was variable around a mean of $1 \mathrm{~min}$.

On each of the next 22 days, they received facilitation training in which $B$ and $P$ served as target keylights for the $T, N$, and $L$ facilitators. Each session contained 12 nonreinforced presentations each of $B$ and $P$, together with 12 reinforced presentations each of $B$ and $P$ in the presence of one of the facilitators. For half of the animals, $T$ served as the facilitator for $B$ and $N$ as the facilitator for P; for the other half of the animals, the auditory stimuli were interchanged. In addition, each session contained six reinforced presentations of each keylight in the presence of the flashing of the houselight. All keylight presentations were $5 \mathrm{sec}$ long; all diffuse stimuli were $15 \mathrm{sec}$ long. On compound trials, the keylight was present during the final $5 \mathrm{sec}$ of the diffuse stimulus. Food presentation consisted of the 5-sec availability of grain.

On each of the next 4 days, the birds received a treatment designed to modify one of the target keylights. Half of the birds in each group received 24 nonreinforced presentations of $B$ in the presence of $L$, whereas the other half of the birds received 24 nonreinforced presentations of $P$ in the presence of $L$.

The white (W) keylight was prepared to be the transfer target over the next 3 days. On the first day, each bird received 36 rein- 
forced 5-sec presentations of $W$. On the following 2 days, they received 36 nonreinforced presentations of $W$. Previous experiments (e.g., Rescorla, 1985) have shown such training and extinction of a keylight to generate a sensitive target for transfer of facilitation.

On the next day, the animals received a test session. That session began with three nonreinforced presentations of $W$ alone. It continued with the intermixing of four nonreinforced presentations each of $\mathrm{W}$ alone and in compound with each of the auditory stimuli. The issue of interest is whether transfer of the auditory stimuli will be differential depending on the treatment of their targets in the previous phase of the experiment.

On the next day, the animals received a test session of the original targets. That session began with two nonreinforced presentations each of $B$ and $P$ and continued with four each of $B$ and $P$ alone and in compound with $L$. The intention of this test was to verify that the differential treatment of the targets had successfully modified them.

\section{Results and Discussion}

Acquisition of facilitation proceeded smoothly. On the final day of training, the mean rates of keypecking were $18.9,135.1$, and 111.7 during the keylights alone and in conjunction with the auditory and visual facilitators, respectively. Although the noise was numerically more successful than the tone as a facilitator, there were not reliable differences as a function of the identity of the keylights or the auditory facilitators. However, both the auditory and the visual facilitators augmented responding to the keylights [Wilcoxon $T \mathrm{~s}(16)=0, p<.01$ ].

Over the course of nonreinforced presentation of one keylight during the light, responding dropped rapidly. On the final day of this differential treatment, the mean rate of pecking to the keylight during $\mathrm{L}$ was 7.9 responses per minute. Autoshaping to the transfer target, $\mathrm{W}$, proceeded smoothly.

The data of most interest, from the transfer test, are shown in Figure 2. Because there were no reliable differences as a function of the identity of the stimuli, that figure combines the results across different facilitators. It displays the rates of pecking separately for the transfer target presented alone and in combination with the diffuse

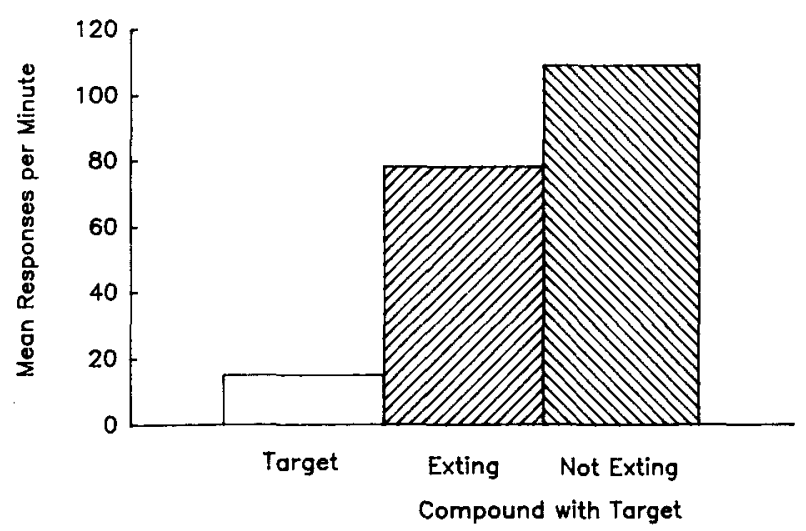

Figure 2. Transfer test results from Experiment 1 showing responding during a target keylight when presented alone and in the presence of diffuse facilitators whose own targets had elsewhere been either extinguished or not. facilitators whose own target had been extinguished or not. It is clear that both auditory facilitators successfully transferred to the test target. However, the magnitude of the transfer was less for the facilitator whose target had been extinguished. Pecking to $\mathrm{W}$ was greater during both the extinguished $[T(15)=1, p<.01]$ and the nonextinguished $[T(15)=0, p<.01]$ facilitator, compared with that to $W$ alone. Of more interest, the extinguished facilitator promoted less keypecking than did the nonextinguished facilitator $[T(12)=8, p<.02]$.

During the second test, each original training target was presented alone and in compound with the light facilitator. That test showed evidence that separate nonreinforcement of the target during $\mathrm{L}$ had undermined responding to that target. Mean responding to the extinguished target was similar whether it was presented alone $(10.3 \mathrm{per}$ minute) or in compound with $\mathrm{L}$ ( 7.4 per minute). By contrast, the nonextinguished target continued to be facilitated by $\mathrm{L}$. The mean response rates to the nonreinforced target were 24.6 and 57.4 per minute when it was presented alone and in compound with $L$, respectively [T(13) $=4, p<.01]$. Comparison with the preextinction levels of responding suggests that the intervening treatment had also reduced the ability of $\mathrm{L}$ to promote responding to the nonpresented target. Although the appropriate comparison is not available in the present experiment, that outcome would be expected on the basis of prior findings on the extinction of facilitation (e.g., Rescorla, 1986a).

These results suggest that the state of the original target for a facilitator is important for the ability of that facilitator to transfer to another target. Extinction of the original target in the presence of one facilitator reduced the ability of another facilitator of that target to transfer. A possible interpretation of this result is that the original training target is somehow involved in the transfer of a facilitator to a new target.

\section{EXPERIMENT 2}

Experiment 2 investigated the effect of target stimulus manipulation on the transfer of conditioned inhibition. The design was analogous to that of Experiment 1. Two auditory stimuli each served as a conditioned inhibitor for its own excitatory keylight. In addition, a diffuse visual stimulus was trained as a conditioned inhibitor for both those keylights. Then, one of the keylights was repeatedly reinforced in the presence of the visual inhibitor in an attempt to reduce the degree to which that keylight was inhibited. Finally, both auditory inhibitors were tested for transfer to a third keylight target. The issue of interest is whether successful transfer of an inhibitor is affected by this manipulation of its target.

\section{Method}

Subjects and Apparatus. The subjects were 15 female pigeons with similar histories to those used in Experiment 1 and maintained in the same manner. The apparatus was the same as that in Experiment 1 . The three keylight stimuli used in Experiment 2 were two colors, blue (B) and yellow (Y), and the grid pattern (P). Blue and 
yellow occupied the bottom portion of the key; P occupied the top portion. The diffuse stimuli were the same $T, N$, and $L$, as used in Experiment 1.

Procedure. The birds received initial conditioned inhibition training for 20 days. During each day, they were exposed to $125-\mathrm{sec}$ reinforced presentations each of $B$ and $Y$. In addition, they received 12 nonreinforced presentations of $B$ in combination with one of the auditory stimuli and 12 of $\mathrm{Y}$ in combination with the other. For 8 animals, $B$ was presented together with $N$, and $Y$ with $T$; for 7 animals, the roles of the auditory stimuli were reversed. In addition, each animal received six nonreinforced presentations of each keylight in compound with $\mathrm{L}$. Throughout this and all subsequent phases, the diffuse stimuli were $15 \mathrm{sec}$ long, the keylights $5 \mathrm{sec}$, and, on compound trials, the keylights occupied the final $5 \mathrm{sec}$ of the diffuse stimulus. All food presentations were $5 \mathrm{sec}$; the ITI was variable around a mean of $1 \mathrm{~min}$.

On the next 5 days, the $P$ keylight was prepared for transfer of the inhibitors. On each of the first 2 days, the animals received 36 reinforced $5-\mathrm{sec}$ presentations of $P$. On each of the next 3 days, they received 36 nonreinforced presentations of $P$.

On the next 3 days, the original target keylights were manipulated. Each day, all animals received 24 presentations each of $Y$ and $B$ with $L$. In a manner counterbalanced with regard to previous group assignments, 8 animals had presentations of LY followed by food and presentations of LB nonreinforced, whereas 7 animals had the reverse treatment.

On the following day, the animals received a transfer test with $P$. The session began with 12 reinforced presentations of $P$ and continued with eight presentations each of P, TP, and NP. Separate presentations of $P$ terminated in food, whereas presentations in compound with an auditory stimulus did not. On the following day, the birds were returned to the original conditioned inhibition training procedure, in order to assess the impact of the target manipulation on the ability of the various inhibitors to act on their original targets

\section{Results and Discussion}

Initial acquisition of conditioned inhibition proceeded smoothly. As in Experiment 1, the noise was numerically, but not statistically, more effective than the tone. On the final day of conditioned inhibition training, the mean rates of responding were $153.8,21.2$, and 11.9 during the keylights alone, combined with the auditory inhibitors, and combined with L, respectively. Differential reinforcement of the target stimuli during the light rapidly led to discriminative performance. On the final day of that training, the mean rates of responding to the reinforced and nonreinforced keylights were 127.9 and 2.5 , respectively.

The data of most interest, from the transfer test of the auditory inhibitors with $\mathrm{P}$, are shown in Figure 3 . Because there were no reliable differences as a function of stimulus identity, the data are combined so as to present separately responding during the target alone and when in compound with the auditory inhibitor whose target had been reinforced or nonreinforced. It is clear that both auditory stimuli successfully transferred their inhibition to P. Responding was greater during the keylight alone than when it was compounded with either inhibitor $[T \mathrm{~s}(14)<14, p \mathrm{~s}<.05]$. Of more interest, the inhibitor whose target had been reinforced during the light transferred less well. Responding was greater to the transfer target during that inhibitor than during the inhibitor whose

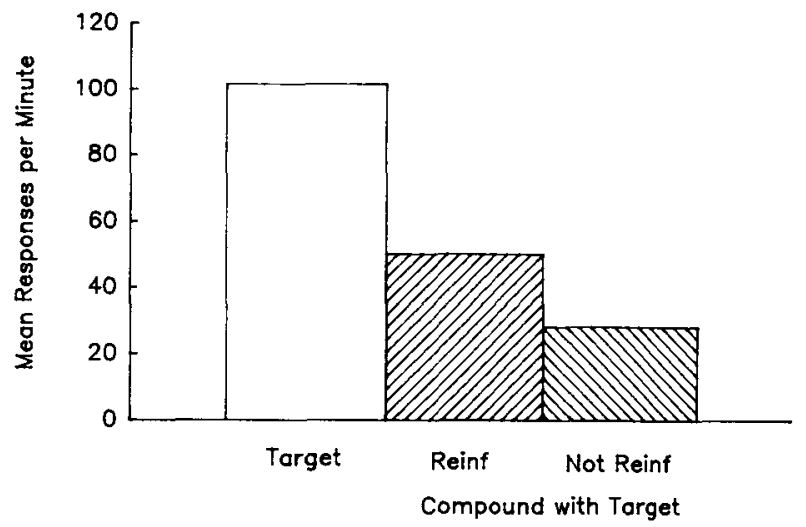

Figure 3. Transfer test results from Experiment 2 showing responding during a target keylight when presented alone and in the presence of diffuse inhibitors whose own targets had elsewhere been either reinforcer or not.

target had not been reinforced $[T(13)=9, p<.01]$. Thus, manipulation of the original target of an inhibitor had an effect similar to manipulation of the original target of a facilitator-it undermined transfer to a new target.

The second test, during which the animals were returned to their original inhibition training regime, confirmed that the treated target keylights had been reduced in their ability to be inhibited. During that test, the targets did not differ in their response rates when presented alone; the rate of responding during the reinforced keylight was 161.3 per minute, whereas that during the nonreinforced keylight was 155.3 per minute $[T(14)=40$, n.s.]. However, these keylights were quite different in the degree to which they could be depressed by their auditory and visual inhibitors. Not surprisingly, the animals responded at a higher rate to the reinforced keylight than to the nonreinforced keylight during the light [72.6 vs. 2.9 per minute; $T(15)=0, p<.011$. Of more interest, the keylight reinforced during the light was also less successfully inhibited by its original auditory inhibitor [96.7 vs. 33.9 responses per minute; $T(15)=0, p<.01$ ]. That reduced ability of one diffuse stimulus to inhibit its own training excitor verifies the success of the manipulation. It suggests the possibility that transfer of that inhibition might be mediated by the ability of the inhibitor to act on its own excitor.

\section{GENERAL DISCUSSION}

These results show a specificity of action of conditioned inhibitors and facilitators to their original training targets. The manipulation of a target stimulus had a specific adverse effect on the operation of the modulator with which it had been trained. It is clear that, despite substantial transfer to a new target, the animal has encoded which modulator was trained with which target. 
Indeed, the procedures used in the present experiments bear more than a passing resemblance to experiments that are designed to show the presence of a within-compound association between two stimuli. There is good evidence for many compound stimulus situations that jointly presented stimuli become associated with one another. A popular technique for demonstrating the existence of such associations is to separately treat one of the stimuli after their joint presentation and then inspect the other stimulus for changes. Using such a logic, a number of investigators have previously shown that conditioned inhibitors become associated with their targets (e.g., Cunningham, 1981; Rescorla, 1982; Speers, Gillan, \& Rescorla, 1980; Williams \& Overmier, 1988). It is less common to find demonstrations of a similar sort for conditioned facilitators.

Therefore, one potential interpretation of the present data is that manipulating the associative strength of the target trained with a modulator in turn affects the associative strength of the modulator via modulator-target associations. That interpretation receives support from previous work in which localized visual stimuli trained as inhibitors had their ability to transfer undermined by direct excitatory training (e.g., Rescorla, 1985). However, there is reason to doubt that variations in the excitatory strength of the diffuse modulators used here have a similar effect. Quite a number of experiments have failed to find any substantial effect on either diffuse inhibitors or diffuse facilitators when their excitatory properties are manipulated by direct pairings with the US (e.g., Rescorla, 1985, 1986a). Such diffuse stimuli do not evoke directed pecking when they are made excitatory by pairing with the US, nor do such treatments carried out after training appear to affect their ability to modulate other stimuli. Consequently, it seems likely that the manipulations conducted here on the targets of those diffuse modulators act in some manner other than by alteration of the Pavlovian excitation of the modulators.

One possibility that seems attractive is that a portion of the action of a modulator is on the association between the A target and the US. Transfer to B then might be partially accomplished by generalization between the A-US and the B-US association. Manipulations of the A-US association, such as its extinction during another facilitator or reinforcement during another inhibitor, could modify the ability of its original modulator to act on that A-US association. As a result, those manipulations would also modify the ability of the modulator to transfer to B. On the other hand, the failure of the present manipulations to completely eliminate transfer suggests that it is not mediated exclusively by the A-US association.

The view that a modulator may partially act on a target-US association has been suggested in several forms by Holland $(1983,1989 a)$. It has also been advocated as an account for a related paradigm in instrumental training. For instance, Rescorla (1990) has recently suggested that a discriminative stimulus in instrumental learning may act on the response-outcome association. He has presented various types of evidence that the stimulus modulates performance by controlling that association.

These results also make less attractive an account of specificity of a modulator to its target in terms of configural cues. According to that view, the animal solves facilitation and inhibition problems like those posed here by treating the TB, NP, LB, and LP stimuli as configural stimuli. In that case, the effects of manipulating LB on $T$ 's ability to modulate a transfer stimulus, $W$, would involve the $\mathrm{LB}$ configural cue modifying the TB configural cue in such a way that the TW configural cue elicited different responding. Such a multistep process seems to strain the utility of a configural view.

Indeed, one recent attempt to place configural cues at the center of complex discriminations (Pearce, 1987) explicitly denies the possibility of such passing along of generalization from one configuration to another. According to that theory, responding to TW and NW occurs only by virtue of their generalization from the associative strengths actually conditioned to TB and NP, respectively (as well as from W). Although manipulations of LB and LP generalize to TB and NP, and therefore affect the responding they control, that generalized strength does not in turn affect the degree to which they generalize to TW and NW. A simulation of that theory verifies that in the present procedures the associative strength directly conditioned to TB and NP is identical at the time of the transfer test. Hence, this version of a configural model fails to predict the outcomes of the present experiments.

One might be able to incorporate the present data by elaborating on a configural model to allow for "mediated stimulus generalization." For instance, Pearce (1987) discusses the possibility that conditioning to A might generalize to $B$ based on the joint similarity of $A$ to $A B$ and of $\mathrm{AB}$ to $\mathrm{B}$, to the degree that an $\mathrm{AB}$ compound is represented in memory. Such a modification might deal not only with the present data but also bring such phenomena as sensory preconditioning within reach of a configural model. However, it raises serious difficulties of its own. For instance, the ability of the Pearce model to explain the low level of responding to $B$ after an $\mathrm{A}+/ \mathrm{AB}+$ blocking procedure depends on the assumption that only the strength directly conditioned to $A B$ (as distinct from that generalized to $A B$ from $A$ ) generalizes to $B$. Blocking occurs because prior training of $A$ prevents $A B$ from acquiring direct associative strength and so limits the strength that can generalize to $B$ in testing. However, permitting the conditioning of $A$ to generalize to $B$ through the mediation of $A B$ would severely undermine that blocking. Indeed, it is not obvious what set of specific assumptions would permit a configural model both to preserve its prediction of blocking and to allow "mediated generalization" of the sort required by the present data.

In sum, the present results clearly demonstrate that at least some of the action of modulators is specific to their 
original training targets. They also suggest that this specificity is not attributable to configural stimuli.

\section{REFERENCES}

Cunningham, C. L. (1981). Associations between the elements of bivalent compound stimulus. Joumal of Experimental Psychology: Animal Behavior Processes, 7, 425-436.

Holland, P. C. (1983). "Occasion-setting" in Pavlovian feature positive discriminations. In M. L. Commons, R. J. Herrnstein, \& A. R. Wagner (Eds.), Quantitative analyses of behavior: Volume IV. Discrimination processes (pp. 183-206). Cambridge, MA: Ballinger.

Holland, P. C. (1985). The nature of conditioned inhibition in serial and simultaneous feature negative discriminations. In R. R. Miller \& N. E. Spear (Eds.), Information processing in animals: Conditioned inhibition (pp. 267-297). Hillsdale, NJ: Erlbaum.

HollaND, P. C. (1989a). Acquisition and transfer of conditioned discrimination performance. Journal of Experimental Psychology: Animal Behavior Processes, 15, 154-165.

Holland, P. C. (1989b). Feature extinction enhances transfer of $o c-$ casion setting. Animal Learning \& Behavior, 17, 269-279.

Holland, P. C. (1989c). Transfer of negative occasion setting and conditioned inhibition across conditioned and unconditioned stimuli. Journal of Experimental Psychology: Animal Behavior Processes, 15, 311-328.
Pearce, J. M. (1987). A model of stimulus generalization for Pavlovian conditioning. Psychological Review, 94, 61-73.

REsCoRla, R. A. (1982). Some consequences of associations between the excitor and the inhibitor in a conditioned inhibition paradigm. Journal of Experimental Psychology: Animal Behavior Processes, 8, 288-298.

Rescorla, R. A. (1985). Inhibition and facilitation. In R. R. Miller \& N. E. Spear (Eds.), Information processing in animals: Conditioned inhibition (pp. 299-326). Hillsdale, NJ: Erlbaum.

ResCorla, R. A. (1986a). Extinction of facilitation. Journal of Experimental Psychology: Animal Behavior Processes, 12, 16-24.

RescorLA, R. A. (1986b). Facilitation and excitation. Journal of Experimental Psychology: Animal Behavior Processes, 12, 325-332.

ResCorla, R. A. (1990). Evidence for an association between the discriminative stimulus and the response-outcome association in instrumental learning. Joumal of Experimental Psychology: Animal Behavior Processes, 16, 326-334.

Speers, M. J., Gillan, D. J., \& Rescorla, R. A. (1980). Withincompound associations in a variety of compound conditioning procedures. Learning \& Motivation, 11, 135-149.

Williams, D. A., \& Overmier, J. B. (1988). Some types of conditioned inhibitors carry collateral excitatory associations. Learning \& Motivation, 19, 345-368.

(Manuscript received September 8, 1990; revision accepted for publication December 12, 1990.) 